\title{
La psychiatrie coloniale en Nouvelle-Calédonie
}

\section{Yoram Mouchenik}

\section{OpenEdition \\ Journals}

Édition électronique

URL : http://journals.openedition.org/jso/1566

DOI : $10.4000 /$ jso. 1566

ISSN : 1760-7256

\section{Éditeur}

Société des océanistes

\section{Édition imprimée}

Date de publication : 1 décembre 2001

Pagination : 109-119

ISSN : 0300-953x

\section{Référence électronique}

Yoram Mouchenik, "La psychiatrie coloniale en Nouvelle-Calédonie », Journal de la Société des

Océanistes [En ligne], 113 | Année 2001-2, mis en ligne le 01 décembre 2001, consulté le 01 mai 2019 URL : http://journals.openedition.org/jso/1566 ; DOI : 10.4000/jso.1566 


\title{
La psychiatrie coloniale en Nouvelle-Calédonie
}

par

\author{
Yoram MOUCHENIK *
}

\section{RÉSUMÉ}

La psychiatrie coloniale en Nouvelle-Calédonie est indissociable de l'action des missions dans le champ de la santé des indigènes et de l'ethnopsychologie kanak du pasteur-ethnologue Maurice Leenhardt. La théorisation d'une "pensée mythique " chez les Mélanésiens sera d'un grand secours pour les élaborations d'une psychiatrie coloniale en peine de caution ethnologique et préférant se réfugier auprès de Leenhardt plutôt que d'utiliser les travaux contemporains sur la NouvelleCalédonie en anthropologie sociale et en ethnomédecine. Le présent article propose un panorama et une réflexion sur la place de ces différents acteurs.

Mots-clef : Nouvelle-calédonie, Missions, ethnologie, psychiatrie, ethnopsychologie.

La psychiatrie coloniale en NouvelleCalédonie est indissociable de l'histoire de l'assistance sanitaire, de la place de la santé dans les stratégies missionnaires et des développements de l'ethnologie du pasteur Maurice Leenhardt. L'ethnopsychologie de la personne kanak chez Maurice Leenhardt participe largement aux conceptions essentialistes de la psychiatrie coloniale qui singularise un psychisme et une psychopathologie kanak. Nous aborderons successivement ces différents aspects en soulignant leur continuité et leur complémentarité dans une conceptualisation psychiatrique de l'indigène,

\begin{abstract}
Colonial psychiatry in New Caledonia is indissociable from the missions medical work for the natives and the ethnopsychology of the Pastor-ethnologist Maurice Leenhardt. His " mythical mind" theory about Melanesians was of a big help for the construction of a colonial psychiatry, looking for an ethnological background with Maurice Leenhardt rather than using contemporary researchs in social anthropology and ethnomedecine. This article describes a panorama with such different actors.
\end{abstract}

Key-words: New Caledonia, Missions, ethnology, psychiatry, ethnopsychology.

particulièrement figée depuis des décennies avec la caution ethnologique d'autorité conférée par les travaux de Maurice Leenhardt. Nous ne traiterons pas ici de la clinique et des évolutions de ces dix dernières années qui sont l'objet d'un travail spécifique ${ }^{1}$.

\section{L'« assistance sanitaire indigène "}

L'« assistance sanitaire indigène » ne connaîtra pas en Nouvelle-Calédonie les mêmes développements qu'elle aura dans d'autres colonies.

* Psychologue-clinicien, Docteur en anthropologie de l'EHESS.

1. $C f$. la thèse d'anthropologie de l'auteur (Mouchenik, 2000). 
Dès 1912, Reboul et Régis, dans leur rapport au congrès des Aliénistes et Neurologistes de Langue Française à Tunis, préconisaient la création pour chaque colonie d'un « réseau d'assistance psychiatrique » (Collignon, 1978 : 134). En Afrique, au début du siècle, les médecins pastoriens prennent pour cible la maladie du sommeil, qui prend des formes épidémiques souvent mortelles pouvant décimer les populations colonisées et les colons. En Nouvelle-Calédonie, la lèpre, répandue dans des proportions très importantes, ne suscitera pendant longtemps que des demimesures impropres à enrayer la maladie dont la gestion est surtout confiée aux missions. En dehors de la ville de Nouméa, des centres de colonisation et de « la pénitentiaire », le monde rural kanak est quasiment laissé sans assistance médicale pendant un siècle, alors que la ville de Nouméa est dotée de deux hôpitaux dès la fin du siècle dernier et d'un Institut de microbiologie en $1913^{2}$. Les médecins des troupes coloniales qui remplacent ceux de la marine à partir de 1890 prennent peu en charge la population indigène. Les premières mesures visent à combattre la lèpre en exilant et stigmatisant les malades avec : «[...] des mesures sanitaires coercitives et non curatives [...]» (Lepoutre, 1997 : 78). Les médecins résidents mis en place pour les Îles Loyauté exercent des fonctions de magistrat, d'administrateur et de contrôle qui laissent très peu de place à l'exercice directe de la médecine. Précurseur d'un maillage sanitaire de la colonie, ce sont les missions qui développent et assurent l'assistance sanitaire indigène, l'administration n'exerçant qu'une lointaine tutelle. Nous illustrerons la place de la santé dans les stratégies missionnaires en soulignant les différences sur fond de rivalité nationale entre les catholiques et les protestants. Ces derniers formeront rapidement un clergé indigène et feront appel à des infirmières professionnelles et diplômés plutôt qu'à de bonnes volontés qui se forment à la tâche comme ce sera souvent le cas chez les catholiques. Cependant, sœurs missionnaires catholiques, demoisellesinfirmières protestantes et femmes de pasteur ont la charge principale de l'« assistance sanitaire indigène ", rôle important, mais subalterne qui ne fait pas partie des tâches nobles de l'évangélisation dévolue aux hommes. Cette « valence différentielle » des attributions suivant le genre et la sphère privée ou publique est largement soulignée dès le début de l'évangélisation au Vanuatu par Margaret Jolly (1991) et apparaît comme une constante dans la conquête missionnaire en Océanie.

\section{Les Maristes, les Søurs et la santé}

Nous nous arrêterons plus particulièrement sur la place de la santé dans les stratégies missionnaires maristes à travers les archives ${ }^{3}$. Sachant que la médecine coloniale et militaire bénéficie surtout aux colons, aux fonctionnaires et à la "pénitentiaire ", les congrégations religieuses sont les premiers acteurs de l'assistance sanitaire ${ }^{4}$ aux indigènes. Les Sœurs, soignantes ou plus tard infirmières en forment le noyau historique. Dans le partage des rôles, les Sœurs prennent souvent en charge la maladie, sans formation et sans expérience. À Ouvéa, par exemple, Sœur Marie-Sébastien 5 fait fonction d'infirmière sans qualification à partir des années vingt en se formant à la tâche auprès des lépreux.

L'intervention des Maristes est particulièrement importante en Nouvelle-Calédonie où ils précèdent, accompagnent et encouragent la colonisation française comme rempart contre le protestantisme anglais : «Leur principal représentant, Mgr Douarre, retourne en France, en 1848 , pour plaider auprès de Guizot la cause de l'annexion. Cette terre, disait-il, deviendra protestante si la France ne se dépêche pas d'en prendre possession. » (Merle, 1995 : 37)

La Société de Marie est créé en 1816. C'est parce qu'elle accepte l'évangélisation d'une partie de l'Océanie que la jeune société obtient en 1836 une reconnaissance rapide de la Sacrée Congrégation de la Propagande de la foi à Rome, qui va permettre son essor (Immacolata Occorsio, $1988: 18$ ). Rapidement les pères maristes en Océanie demanderont le renfort des religieuses pour ce qui concerne les tâches de santé de la population et l'éducation des filles : « Les Maristes agiront comme Marie d'une façon discrète : Ignoti et quasi occulti in hoc mundo, préciseront leurs règles. C'est pour une grande efficacité que l'apostolat doit rester caché dans un monde qui se méfie des religieux » (Immacolata Occorsio, $1988: 11)$.

Françoise Perroton encore laïque, première missionnaire de l'Océanie centrale, s'embarque le 15 novembre 1845 sur l'Arche d'Alliance pour

2. Un laboratoire de bactériologie fonctionnait déjà à Nouméa en 1901 (rapport du Docteur Noc, Annales d'hygiène et de médecine coloniale, t.7, 1904 : 477-490, cité dans la bibliographie médicale du Docteur Zeldine, Nouméa).

3. Je remercie Sœur de Mijolla, historienne des Sœurs missionnaires de la Société de Marie et infirmière dans la Province Nord pour ses précieuses informations qui m'ont permis d'accéder à certains travaux sur ce sujet.

4. Marie Lepoutre (1997) a particulièrement étudié l'histoire de l'implantation de l'assistance sanitaire à Lifou.

5. Communication personnelle de Sœur Marie Saint-Sébastien, née en 1905 (Saint Louis, janvier 1998). 
Wallis et ensuite pour Futuna. La présence des Sœurs en Nouvelle-Calédonie pour les tâches de santé et d'éducation est rapidement perçue comme indispensable: " Deux religieuses placées à la Conception feraient un bien inappréciable, soit pour le soin des malades, soit pour former les personnes de leur sexe aux occupations qui leur conviennent, les instruire, les relever petit à petit de l'état de dégradation où le paganisme les a fait descendre. Sans l'éducation des filles, qui n'est possible à la Nouvelle-Calédonie que par des religieuses, il faudra renoncer à former la famille, et par conséquent priver les indigènes du bienfait d'une vraie civilisation » (Nos Pionnières I, $1973: 48)^{6}$.

En Nouvelle-Calédonie, Monseigneur Douarre, qui réinstalle une mission en 1851, fait appel aux Tierçaires. Trois Sœurs débarquent au Mont Dore le 2 décembre 1858 dont Sœur du Bon Secours (Clothilde Viannay) : «[...] instruite et bien formée, c'est une très bonne infirmière qui sait saigner ${ }^{7}$ et soigner les malades " (de Mijolla, 1980: 55).

$\mathrm{Au}$ mois de juin de la même année, le père Bernard, qui a connu Françoise Perroton à Wallis, réclame des Sœurs pour l'île d'Ouvéa (de Mijolla, 1980). Il avait été précédé dans ses demandes par le père Viard à Wallis : « Si parmi ces personnes que vous pourriez nous envoyer quand vous le jugerez à propos, il s'en trouvait une qui sût traiter les malades et en prendre soin, elle gagnerait facilement l'affection de toute l'île; elle aurait un empire souverain sur les cœurs de nos néophytes et par sa douceur et ses soins elle contribuerait puissamment à leur faire chérir une religion qui leur rend de si grands bienfaits » (Nos pionnières I, $1973: 11$ ).

L'impact des Sœurs qui soignent est particulièrement important en Océanie : «[...] Cependant ce qui fait le plus de bruit dans le pays, c'est la médecine de la Révérente Mère Xavier : elle fait courir tout le monde et on dit : pour le coup, voilà une femme savante qu'on nous a envoyée de chez les Blancs : elle guérit tous les Tongiens de toutes les maladies. [...]» (Nos pionnières I, 1973 :153). Ou bien : « Sœur Marie Rose [...] fait merveille : avec peu de remèdes, elle ressuscite presque les morts. On a tellement confiance en elle que l'on apporte les malades de tous les coins de l'île, et quand elle a tâté, palpé, graissé, mijoté, elle leur dit, qu'il n'y a pas de danger et déjà, ils sont à moitié guéris. [...], elle nous fait part de ses bénéfices qui sont encore assez forts quand les naturels avaient pensé que la maladie était grave. Depuis six mois, nous avons reçu trois cochons vivants et plusieurs paniers d'ignames " (Nos Pionnières I, $1973:$ 180) ${ }^{8}$.

La constitution d'une congrégation des Sœurs missionnaires ${ }^{9}$, qui prendront en charge nombre des tâches de santé et d'éducation, sera longue et difficile, avec différentes étapes et des divergences importantes avec les pères Maristes, peu pressés de donner un statut aux Sœurs corvéables à merci. Le père Poupinel sera la cheville ouvrière de cette construction. Comme visiteur des missions maristes d'Océanie, il est très vite sensible à la mauvaise situation des Sœurs complètement dépendantes de l'autoritarisme des pères missionnaires tant qu'elles ne relèvent pas d'une congrégation qui leur soit propre. La tentative de structurer cette congrégation autour de la supérieure de Notre-Dame-des-Missions aboutit à une rupture avec les pères Maristes après une longue période de divergences sur le fonctionnement de la congrégation. Beaucoup de Sœurs missionnaires travaillant sur le terrain refuseront l'allégeance demandée par NotreDame-des-Missions avec des règles contraignantes qui menaçaient de les empêcher de poursuivre leur travail missionnaire: grillage, vie collective cloîtrée - alors qu'elles sont dispersées - temps de prières et de méditation quotidien imposé. Les ponts seront rompus entre la Société de Marie et Notre-Dame-des-Missions dès 1869 et la congrégation ne sera plus représentée en Nouvelle-Calédonie à partir de 1871 (de Mijolla, 1980). En 1875 sont formées à SaintLouis les trois premières Petites Filles de Marie, premières Sœurs indigènes destinées à seconder les Sœurs françaises ou anglaises et qui essaimeront dans toute la Calédonie : «[...] Chose qui vous étonnera davantage: je vais donner un habit bleu à trois jeunes filles noires que je destine à seconder S.M. de la Croix. Depuis un an elles me demandent de se consacrer à la mission, comme novices tierçaires. Comme elles ne feront pas de vœux, cela n'est pas dangereux. [...]» (Nos Pionnières IV, $1975: 22)^{10}$.

Ce projet était déjà en germe chez le père Poupinel dès $1865:$ « Mon intention n'est pas de

6. Lettre $\mathrm{n}^{\circ} 51$ du P. Poupinel (Sydney) au P. Favre (Lyon), 9 décembre 1857, APM OP 418.

7. Les sœurs exportent une médecine des humeurs et des pratiques opératoires qui ne sont pas sans rapport avec les représentations du corps sur les Îles Loyauté, les humeurs (sang) et une médecine évacuante (scarification, purge).

8. Lettre $\mathrm{n}^{\circ} 178$ de S.M. de la Miséricorde (Wallis) à Yardin (Lyon), 5 février 1860, ASMSM.

9. Après l'échec de Notre-Dame-des-Missions sera constitué le Tiers Ordre de Marie, mais il faut attendre 1932 pour l'approbation des Sœurs Missionnaires de la Société de Marie comme congrégation de droit pontifical (de Mijolla, 1980).

10. Lettre $\mathrm{n}^{\circ} 658$, Vitte (Nouméa) à Germain (Lyon), 3 septembre 1875, APM ONC 418.1 
demander que ces jeunes filles océaniennes soient admises dans la congrégation sur le même pied que les Sœurs préparées en France, françaises ou anglaises. Pour plusieurs raisons, au moins jusqu'à expérience faite, il vaudrait mieux, à mon avis, qu'elles formassent une classe séparée et inférieure, comme des tierçaires par exemple, et dont les vœux ne seraient qu'annuels[...] c'est qu'on doit encore les considérer en masse comme des néophytes et d'une espèce plus faible : c'est que si les océaniennes étaient admises au même rang que les françaises et les anglaises, les premières (les françaises) en seraient peu formalisées, je crois, mais il n'en serait peut-être pas de même des secondes " (Nos Pionnières II, 1973 : 204) ${ }^{11}$. Ultérieurement les « Petites Filles de Marie » n'auront plus cette place subordonnée. Dans la période contemporaine, des Sœurs infirmières diplômées sont salariées par les dispensaires des Îles Loyauté.

\section{Les missions protestantes et la santé 12}

Le professionnalisme de l'«assistance sanitaire indigène » caractérise sa mise en œuvre par la mission protestante. Les "demoiselles missionnaires » recrutées par la Société des Missions Évangéliques de Paris sont diplômées et expérimentées. Elles interviennent directement auprès de la population, mais forment également les femmes de pasteurs kanak aux soins et à l'obstétrique (Salomon, 1999). Reconnues par l'administration et les rares médecins coloniaux, les «demoiselles missionnaires» infirmières sont à plusieurs reprises récompensées par la «médaille d'honneur des épidémies» (1935), par des " témoignages officiels de satisfaction » (1960) ou par une nomination au grade de "Chevalier de l'ordre de la Santé Publique» (1961). Dans les années trente, l'administration coloniale subventionne en partie le recrutement d'infirmière missionnaire ainsi que leurs frais de voyage jusqu'à Nouméa ${ }^{13}$. C'est au nom du professionnalisme des «demoiselles » que la mission protestante proposera de prendre en charge l'« assistance sanitaire indigène » à Ouvéa au début des années trente en se démarquant des Sœurs catholiques non formées : «Toute question de dévouement mise à part, et du simple point de vue médical, il nous semble que Mademoiselle Geiser, ayant fait des études spéciales, est plus qualifiée que Sœur Marie SaintSébastien pour s'occuper d'assistance médicale dans une île dépourvue de médecin ${ }^{14}$. La mission médicale protestante sera, quelquefois, un interlocuteur privilégié pour le Service de Santé coloniale. Cette reconnaissance de l'administration ou de la mission n'empêche pas les demoiselles de se sentir isolées et démunies avec un travail considérable. Dans les recommandations adressées en 1963 à une nouvelle missionnaire infirmière sont énumérées en plus du travail médical les tâches suivantes : «[...] visiter inlassablement les villages, [...] encourager les pasteurs, animer nos trois écoles, travailler parmi les femmes et jeunes filles (puériculture, groupe de couture... etc.) $\gg 15$.

Une fois passée la période de conquête évangélique, les missionnaires catholiques et protestants vont davantage se consacrer à contrôler et fidéliser les populations et ils auront un quasimonopole pendant longtemps sur l'enseignement et la santé, presque à l'instar de Fidji où, en 1899 , la mission catholique se voit officiellement confiée les soins à la population : «En 1899, un gouverneur sympathique à la mission catholique ayant été nommé dans cet archipel, elles se voient confier la 'Mission hygiénique' : elles vont dans les villages pour y soigner les malades, jeunes et vieux, et donner aux femmes quelques notions d'hygiène et de puériculture » (de Mijolla, 1980 : 233). Avec le faible maillage sanitaire de la colonie à travers les dispensaires, l'assistance sanitaire se laïcise pour être prise en main par les médecins militaires avant d'être confiée aux civils dans les années 1980. Ces transformations feront l'objet de conventions et les sœurs infirmières seront intégrées et salariées dans ces dispositifs. Les dispensaires serviront de point d'appui pour les rares passages des psychiatres et l'ébauche d'une psychiatrie ambulatoire.

\section{L'ethnopsychologie chez Maurice Leenhardt}

Maurice Leenhardt (1878-1954), pasteurethnologue, occupe la place centrale de l'ethnologie calédonienne. Les convictions de Maurice

11. Lettre $n^{\circ} 401$, Poupinel (Villa Maria) à Yardin (Lyon), 7 novembre 1865, APM OP 418.

12. Nous resterons bref sur l'«assistance sanitaire indigène» de la mission protestante qui a fait l'objet de travaux importants notamment pour Lifou (thèse de Marie Lepoutre, 1997).

13. Comme le précise un courrier du 13 janvier 1937 du Gouverneur de la Nouvelle-Calédonie au pasteur Lacheret, archives de l'Église Évangélique en Nouvelle-Calédonie et aux Îles Loyauté, Nouméa.

14. Courrier du pasteur Vincent (11 février 1932) au Gouverneur Général de la Nouvelle-Calédonie, archives de l'Église Évangélique en Nouvelle-Calédonie et aux Îles Loyauté, Nouméa.

15. Lettre du pasteur Marc-André Ledoux du 10 avril 1963 à Claudine Bieli, archives de l'Église Évangélique en Nouvelle-Calédonie et aux Îles Loyauté, Nouméa. 
Leenhardt font partie intégrante de sa mission évangélique, de son œuvre ethnologique et de son action politique. Après un parcours d'autodidacte, il laisse une œuvre dense et consacrée ; en ethnologie, il sera presque le seul chercheur français à s'intéresser à cette région de la Mélanésie jusqu'aux années cinquante ${ }^{16}$. La rencontre avec Lévy-Bruhl, en 1921, ne marque pas ses débuts ethnographiques, que l'on peut au contraire associer à ses premières années missionnaires (Clifford, 1987 : 13) et à son sentiment de la nécessité d'étudier la "psychologie complète » des Chrétiens mélanésiens (Lettres à ses parents, probablement de 1905, citée par Angleviel (1994: VI). Son intérêt pour les sciences humaines apparaît indissociable de son projet missionnaire. Après une ethnographie minutieuse, qui reste la référence incontournable avec des aspects particulièrement novateurs et précurseurs d'une recherche collective, interactive et " dialogique" 17 , les travaux plus tardifs que nous évoquons ici cherchent d'une façon plus philosophique qu'ethnographique (Naepels, 1996) à définir une essence de la personnalité indigène.

Dans une analyse quasi ontologique, l'être kanak est décrit dans son indifférenciation avec la nature : « Le mélanésien vit indéterminé dans l'enveloppement de la nature. Il ne se répand pas dans la nature; il est envahi par elle. C'est au travers d'elle qu'il se connaît" (Leenhardt, 1944 : 5). De cette participation déjà présente chez Lévi-Bruhl découle la spécificité des fonctions mentales, cognitives et affectives, dans la perception d'un monde sans troisième dimension : " Il n'y a pas de distances entre les gens et les choses ; l'objet adhère au sujet ; des participations imprévues se manifestent dans un monde que l'œil, encore inaccoutumé à saisir la profondeur, ne regarde que sous l'aspect de deux dimensions ${ }^{18} \gg$ (idem : 5). Cette identité avec la nature est un des jalons pour accéder à un « moi psychologique » encore à l'état virtuel dans une « cosmomorphie», précédant une " anthropomorphie » comme dernière étape de transformation pour accéder à l'identité individuelle. À cette étape de son développement ontologique : "Cette identité est vécue par lui, et non perçue. Elle l'empêche de poser une limite entre le monde et lui. Elle facilite les participations et les métamorphoses. Elle prévient tout effort de l'homme pour enfermer en lui l'expérience qu'il a du déroulement de sa propre existence » (idem : 6).

Dans son essence participative du monde minéral, végétal et animal, le Kanak est indifférencié 19 et existe dans les uns ou dans les autres, et non pas dans une conscience personnelle de sujet. Il est de la substance de son environnement et l'igname sacrée, cérémonielle et cultivée, n'est pas un symbole soulignant un dedans et un dehors de l'homme : "Le cycle de l'existence de l'homme est enfermé dans le cycle de l'igname. Et l'expression de ce cycle ne correspond pas à une métaphore, comme on pourrait le penser. $\mathrm{Ce}$ cycle est la projection sur l'igname de l'existence de l'homme. L'homme ignore sa propre existence, il ne peut la saisir » (idem : 8). Dans cette consubstantialité avec la nature, il ne pourrait se concevoir à l'origine de la naissance ${ }^{20}$, la femme comme la terre est à elle-même sa propre fécondité dont l'homme ne serait qu'un fortifiant parmi d'autres : «Le Mélanésien ignore le rôle essentiel de l'homme dans la transmission de la vie [...]. L'époux n'est pas un générateur, il est un roborateur » (idem :11).

Dans la description d'une altérité radicale, l'indigène resterait totalement étranger à qui ne

16. À une époque où, dans d'autres régions de la Mélanésie, des travaux renouvelaient la discipline : « Quand Malinowski était déjà fort célèbre et très lu, quand Hocart publiait des travaux fondamentaux sur Fidji, quand l'anthropologie sociale avait déjà acquis ses titres de noblesse, Leenhardt élaborait en autodidacte son ethnologie missionnaire et inspirée, où les observations ethnologiques souvent bonnes, venaient s'insérer dans une psychologie évolutionniste de la conscience religieuse » (Bensa, $1995: 261)$

17. James Clifford (1980 : 527) souligne l'exceptionnelle originalité de l'entreprise ethnologique de Maurice Leenhardt dans son travail collectif : "The starting point would not be the anthropologist's interpretive descriptions, but rather those of the informant, considered here in the role of indigenous ethnographer $»$.

18. L'idée d'une non-perception de la troisième dimension, la profondeur dans la structuration psychique de l'espace chez les colonisés, avec des explications diverses, est présente chez Maurice Leenhardt et particulièrement répandue pour l'Afrique. Selon Rakowska-Jaillard (1981) le portage au dos du jeune enfant togolais, lui cachant l'espace devant lui, l'empêcherait de structurer la troisième dimension. Je remercie Blandine Bril d'avoir attiré mon attention sur cet auteur (communication personnelle, janvier 2000)

19. Marcel Mauss était plus que sceptique sur des notions de non-individuation et d'indifférenciation : « [...] il est évident, surtout pour nous, qu'il n'y a jamais eu d'être humain qui n'ait eu le sens, non seulement de son corps, mais aussi de son individualité spirituelle et corporelle à la fois » (1960/1938: 335).

20. Devereux (1980 : 77) se posera la question d'une telle représentation chez Malinowski : « Malinowski (1932), discutant la négation par les Trobrianais du rôle du coït dans la fécondation, n'a pas utilisé le mythe, qu'il rapporte pourtant lui-même, de l'eau tombant goutte à goutte dans le vagin d'une femme sur le point d'être fécondée ». 
pourrait le percevoir de l'intérieur ${ }^{21}$. Leenhardt pose les jalons des théorisations qu'il illustre avec la linguistique des langues austronésiennes (1946) et qu'il va longuement développer dans son ouvrage le mieux connu, Do kamo (1947), où il poursuit l'élaboration du fonctionnement mental de la personnalité kanak au travers de la pensée mythique et socio-mythique comme soubassement phénoménologique de sa façon d'être au monde, avec ses corollaires de pensée prélogique, de non-rationalité et de non individuation ${ }^{22}$. Dans cette dernière période Maurice Leenhardt décrit une personnalité kanak sans référence à un contexte. Cette élaboration ${ }^{23}$, qui utilise des arguments tirés de la linguistique qui seront fortement battus en brèche par les travaux ultérieurs ${ }^{24}$, porte la marque d'une anthropologie essentialiste. Elle définit de manière péremptoire la nature « vraie» de l'indigène en soulignant son altérité radicale et sa «mentalité archaïque » (Leenhardt, 1952: 285) dans une « société primitive» ${ }^{25}$. (idem : 289).

À partir des années 1970 et 1980, l'anthropologie sociale d'Alban Bensa, associée aux travaux des linguistes, opérera une rupture en contextualisant la recherche ethnologique.

Notre mise en relief critique rend peu justice à l'œuvre de Maurice Leenhardt d'où sont issues une connaissance et une analyse inégalées du monde kanak ${ }^{26}$. Cependant, dans sa dernière période ${ }^{27}$, elle porte en germe les travers de certains aspects de la future ethnopsychiatrie coloniale ${ }^{28}$.

\section{Psychiatrie « exotique » et ethnomédecine}

La psychiatrie coloniale va puiser à différentes sources en passant par l'ethnologie et les reliquats d'une anthropologie physique. Jusque dans les années 1960, un certain nombre de travaux restent fortement marqués par l'anthropologie physique du XIX ${ }^{\mathrm{e}}$ siècle portant sur la nature même d'une race kanak, qui serait restée à un stade archaïque de l'évolution humaine : «Les caractères physiques des néo-calédoniens ont été comparés et reconnus sur certains points, analogues à ceux des hommes fossiles de la race de Néanderthal ${ }^{29}$. C'est dire qu'ils sont primitifs » (Pales, Chippeaux, Pineau, 1960 : 45) ; ou bien : «Par sa morphologie et son architecture osseuse, le pied des Canaques s'apparente à celui des hommes de Néanderthal. Revêtu de ses parties molles, il évoque par-delà les millénaires, l'image vivante de cette race disparue ${ }^{30} \gg$ (idem : 88). Cette définition d'une altérité radicale de l'espèce kanak ${ }^{31}$ et de sa survivance anachroni-

21. Michel Naepels (1996: 91) souligne à propos de Do kamo la posture particulière de Maurice Leenhardt dans sa définition d'une " expérience kanake du monde ».

22. L'ontologie kanak de Maurice Leenhardt, fortement datée, peut trouver des émules contemporains : « [...] il convient de se référer à une analyse remarquable de Maurice Leenhardt qui, en une vision synthétique, caractérise cette attitude selon les termes suivants : absence de distance entre les gens et les choses. Adhérence du sujet à l'objet. Identité de substance entre le corps humain végétal et animal, que le Canaque 'confond' dans le même flux de vie» (Garelli, 1995 : 267).

23. Jean Guiart se montrera critique d'une vision figée de la personne kanak : « Maurice Leenhardt a cherché comment le primitif, objet de son attention perspicace, structurait sa vie quotidienne et sa société. Pris dans le jeu des idées de son temps, et visant à une explication totale, il ne voulut pas localiser les structures reconnues, cherchant à décrire un mélanésien qui serait de tous les temps et de tous les lieux » (1964:197).

24. Consulter les notes critiques de Jean-Claude Rivierre et Alban Bensa à ce sujet (Bensa, $1995: 248,249,270,271$ ).

25. Adam Kuper explique la longue persistance de cette conception depuis le XIX ${ }^{\mathrm{e}}$ siècle et son usage multiple en ethnologie, sociologie, psychanalyse, sciences politiques, évangélisation par sa «fonction idéologique » irremplaçable (1988: 240).

26. On peut à bon droit penser que l'activité, la persévérance et la longue présence de Maurice Leenhardt à un moment historique charnière ont favorisé la survie et le développement de la population kanak, il apparaît pour certains auteurs comme un précurseur dans le courant de la théologie de la libération.

27. L'immense place de Maurice Leenhardt en ethnologie calédonienne autorise peu des évaluations rétrospectives nuancées de son œuvre, ce qui peut devenir un frein à la réflexion nécessaire sur la discipline et sur ses acteurs.

28. Si l'ethnopsychologie de Maurice Leenhardt est encore utilisée en psychiatrie jusqu'à cette fin de siècle, c'est aussi dans ses aspects novateurs, passant de la " pensée mystique, primitive, pré-logique », en vogue également en psychiatrie coloniale, à la « pensée mythique » dont on ne peut penser qu'elle n'ait de lien avec « la pensée sauvage » de Lévi-Strauss, bien que ce dernier fasse peu référence à l'œuvre de Maurice Leenhardt.

29. Assertion déjà présente dans les travaux sur la Nouvelle-Calédonie du naturaliste-anthropologue suisse, Fritz Sarazin, au début du siècle.

30. Dans les années trente Maurice Leenhardt s'opposera vigoureusement à cette thèse : «Bien plus un livre récent d'anthropologie raconte que le squelette des Néo-Calédoniens comporte des traits plus primitifs que ceux de l'homme de Néanderthal et de tous les hommes fossiles jusqu'ici connus, et les Canaques constitueraient dès lors le rameau le plus primitif de l'humanité actuelle. Et voilà encore un argument où l'on veut trouver une raison à l'extinction de cette race curieuse et périméee » (1931-33:44).

31. On peut faire un prudent rapprochement avec une « raciologie » qui s'exercera antérieurement pour l'Océanie sous la plume de George Montandon (1937) avant que ce dernier ne mettent ses théories au service de la collaboration, pendant l'occupation dans l'Ethnie Française. Dès la fin 1941, il sera attaché en qualité d'ethnologue au Commissariat général aux 
que reste assez proche des commentateurs du $\mathrm{XIX}^{\mathrm{e}}$ siècle tant sur l'anatomie que sur la personnalité ${ }^{32}$. A contrario, de rares médecins ${ }^{33}$ ainsi que le père Lambert ${ }^{34}$ avaient fait quelques notations psychiatriques au siècle dernier. La différence kanak, tellement attestée dans des composantes anatomiques, cognitives et psychologiques ouvre la voie à une élaboration psychiatrique spécifique.

À partir des années 1960, le modèle théorique de la "pensée mythique » ${ }^{35}$ kanak de Maurice Leenhardt sera aisément détournée par une psychiatrie ${ }^{36}$ coloniale en peine de caution ethnologique, le plus souvent à partir des observations des psychiatres militaires expatriés dans l'espace clos de l'hôpital psychiatrique de Nouville, à Nouméa, où sont internés des indigènes. La « pensée mythique » serait à l'œuvre dans la psychopathologie indigène et peut donner lieu à des conceptualisations sur la "folie canaque» (Bourret, Zeldine, 1978 : 549) ou «l'hystérie délirante canaque » (Lachaume, 1987 : 1251). La théorisation médicale est assez pauvre, la pensée mythique kanak spécifique permettant à son tour de justifier une folie kanak sans pour autant définir de « culture bound syndroms » 37 plus précisément.

Selon Poinso et Vedié, en Nouvelle-Calédonie, «Les Européens sont hospitalisés pour des syndromes dépressifs, des tentatives de suicide et des troubles névrotiques. Au contraire, Mélanésiens et Wallisiens sont surtout hospitalisés pour des états psychotiques aigus et chroniques » (1991: 663). Cette division diagnostique défavorable, la psychose pour les indigènes ${ }^{38}$, la névrose pour les Européens, est assez fréquente dans la littérature spécialisée (Selod, 1998) sans que les moyens diagnostiques et théoriques soient très affinés. Les auteurs nous expliquent qu'un traducteur est inutile ${ }^{39}$, - cette affirmation surprend quand on connaît la vitalité des langues parlées en Nouvelle-Calédonie - et ils précisent que la culture protège contre la culpabilité :
«L'absence de tentatives de suicide est également un point que nous avons souligné à plusieurs reprises. La culture mélanésienne par sa vision 'projective' de l'origine des maladies ne favorise pas les sentiments de culpabilité, et ce malgré l'influence de l'église catholique et des missions protestantes » (Poinso, Vedié, 1991: 671). En tâtonnant un peu, les auteurs poussent les Kanak hors du complexe d'Edipe auquel ils n'auraient pas accès: "Nous pensons au contraire qu'il est préférable de poser l'hypothèse que les mécanismes de défense névrotiques sont trop faibles pour être mis en œuvre efficacement. Cela peut s'expliquer par l'éducation tribale qui relègue la triangulation œdipienne au deuxième plan, comme d'ailleurs l'adoption coutumière très fréquente chez les Mélanésiens (ou encore la notion que le 'souffle de vie' provient en réalité du frère de la mère, c'est-à-dire de l'oncle utérin, et non pas du père). Mais la pensée magique, projective, du Boucan nous paraît être un élément prédisposant plus important » (idem: 671). Les auteurs reprennent après Haxaire (1985) et Lachaume (1987) une prédisposition projective : «Un autre aspect de la culture mélanésienne est la facilitation des mécanismes projectifs en rapport avec une pensée mythique et surnaturelle. Pour le Mélanésien, le surnaturel et le mythique sont étroitement présents dans la vie de tous les jours; nulle distance spatiale ou temporelle entre le surnaturel et le réel. La proximité de ces deux mondes permet un passage facile de l'un à l'autre " (Lachaume, 1987 : 1256). La pensée mythique de Leenhardt donnait à penser à un panthéisme et à la nostalgie d'une harmonie avec la nature, perdue pour l'européen, mais conservée par les Kanak. Avec les psychiatres, elle trouve sa version psychopathologique observable à l'hôpital psychiatrique et permettant une catégorisation supplémentaire de l'altérité et du différent.

Ces éléments d'une psychiatrie de l'indigène, en partie issus de l'ethnopsychologie développée

Questions juives avec l'autorité que lui conférait son ouvrage ; Comment reconnaître un juif. En mars 1943, il prend la direction de l'Institut d'études des questions juives et ethno-raciales (Poznanski, 1994).

32. Voir à titre d'illustration L'Ethnographie criminelle dans les colonies françaises (Corre, 1894 : 394-421).

33. «[...] une forme de folie aiguë chez les hommes un peu âgés, [...]» (Vinson, 1858:23) ; consulter également de Rochas (1860).

34. « [...] folie passagère » $(1900: 227-228)$.

35. Pour la psychiatrie la «pensée mythique» de Maurice Leenhardt présente des aspects novateurs plus élaborés que le « primitivisme » et la « mentalité primitive » déjà largement présents en psychiatrie coloniale depuis le début du siècle, voir Porot (1943: 371 et 372$)$.

36. À l'exception notable du mémoire de psychiatrie d'Éric Franchette, (1975) rédigé en France avant son retour en Nouvelle-Calédonie, qui évoque le concept sans l'instrumentaliser directement comme ses collègues, ainsi que la maitrise de psychologie d'Anne-Françoise Laville-Cuenet (1988).

37. Comme par exemple les définit Marie-Rose Moro (1998:15): « [...] nombre de syndromes pourraient être lus de cette façon, c'est-à-dire comme des entités liées à la culture, rendues cohérentes, organisées par la culture ».

38. Thèse précédemment défendue par Zeldine (1981:406).

39. « Le Français est la langue commune, et nous n'avons que rarement recours à un interprète » (idem : 664). 
par Maurice Leenhardt, resteront quasiment inchangés pendant plusieurs décennies. Ainsi, pour Lenormand, qui reprend Leenhardt presque mot à mot: " Physiquement, il [le Mélanésien de l'île de Lifou] ne se différencie pas du monde végétal et minéral auquel il participe et même s'identifie comme partie intégrante d'une même unité mythique du monde. [...] Il ne parvient pas à dégager la notion de sa propre personne en se pensant comme une unité isolable et isolée et même comme fraction d'un ensemble, car il ne peut se concevoir lui-même, sur le plan psychique, que par rapport au monde mythique où il vit et à la société dans laquelle il agit » (Lenormand, 1950 : 64).

Réinterprétant à sa façon les travaux de Maurice Leenhardt, Éliane Métais (1967), qui étudie la croyance sorcière dans une tribu de la Grande Terre, dresse le tableau saisissant d'une personnalité kanak terriblement angoissée, en perpétuel état de crise psychopathologique, et qui lutte contre l'anéantissement psychique par la magie, la religion archaïque et la figuration projective de l'attaque sorcière qui décime la tribu : «Dans la période qui nous préoccupe, tout individu a un psychisme bouleversé et traduit cette angoisse continuelle, sournoise par l'attente d'un châtiment immanquable. Il s'agit d'une véritable psychose de persécution » (1967 : 217). L'intense dramatisation de la description psychologique de la personnalité kanak, submergée par l'angoisse, la désespérance, la dépressivité, la peur et l'effondrement liés à la colonisation, à la destructuration sociale, à la chute démographique et à une incapacité d'adaptation, participent d'une vision d'un psychisme ethnique qui, perdant son cadre de référence traditionnel, ne permettrait la survie du groupe qu'au travers des mécanismes quasi pathologiques de l'interprétation sorcière. Se référant principalement au cahier de doléances du chef Théodore Braïno 40 et aux travaux de Maurice Leenhardt dont elle critique la vision trop harmonieuse du psychisme kanak ${ }^{41}$, l'auteur associe à son point de vue cataclysmique les conclusions étonnantes de son ethnopsychologie kanak : " Enfin, ils manquent de cette vitalité qui conduit les peuples africains à reconstruire pour tout changer; le fond pessimiste, la passivité, l'asthénie que nous venons de montrer et qui les tournent vers une sorte d'attente angoissée d'un avenir tragique, sont tout le contraire du dynamisme propre aux mouvements de libération, d'évasions prophéti- ques politico-religieuses » (idem : 382). Seule la sublimation dans une compétition intellectuelle avec les « Blancs » offrirait : «[...] aux jeunes la possibilité d'éliminer l'asthénie débilitante que la vie en tribu fait régner sur ces membres » (idem : 383). Après avoir longuement décrit le rôle de la colonisation dans une ethnopsychologie kanak pathologique, l'auteur préconise une psychothérapie des tribus ensorcelées par le colonisateur : « Dans le cas de névrose collective, comme celle-ci, nos moyens de prévention, détection et guérison des maladies changent de face; ils doivent être intégrés au réel magique auquel ils s'attaquent. [...] Le meilleur moyen nous paraît être la substitution au devin canaque du médecin blanc dans la pensée indigène » (idem : 309). Le «médecin blanc» devient le : « [...] seul artisan du salut actuellement » (idem: 384). Pour conclure l'auteur conseille de faire soigner les plus atteints dans les infrastructures psychiatriques de la colonie.

C'est aux travaux de Bourret (1981-82) que l'on peut faire remonter les débuts d'une élaboration sur les pratiques médicales et les classifications traditionnelles kanak et d'une ethnomédecine qui trouvera sa théorisation la plus précise et la plus dégagée des poncifs antérieurs dans les travaux de Salomon (1993, 2000). Selon Bourret, Leenhardt et Lenormand sont restés prisonniers l'un et l'autre de leur formation médicale dans leur traduction du vocabulaire anatomique: «[...] les termes recueillis par Leenhardt et Lenormand sont interprétés. Le premier, missionnaire instruit en médecine, le second pharmacien, ayant tous deux étudié selon les méthodes en faveur dans la première moitié du siècle, ont tenté de donner des définitions mélanésiennes des traductions européennes. [...] Une transcription systématique ôte à la nomenclature et à la classification indigène la dimension culturelle indispensable à la compréhension de la connaissance médicale mélanésienne » (1981-82 : 493). Dans ses travaux, Bourret ébauche une typologie des maladies, mais sans les insérer dans la trame symbolique qui leur donne un sens, se rapportant à la production d'une conception indigène de la maladie non rattachée à une vision du monde dont elle n'est que le prolongement.

Le travail princeps de Salomon (1993, 2000) sur le centre-nord de la Grande Terre opère une rupture radicale avec les travaux antérieurs, en réintégrant le point de vue kanak sur la ques-

40. Les accusations du chef adressées au Gouverneur de la colonie s'inscrivent elles-mêmes dans une stratégie politique sous-estimée par l'auteur, comme le souligne Guiart (1967). À une époque, avant l'abolition du code de l'indigénat où, dans la législation, l'accusation de sorcellerie peut permettre de punir et d'exiler des sujets peu dociles.

41. « [...] M. Leenhardt, pour qui l'indigène vit dans un état de paix et presque d'euphorie » (idem : 131). 
tion ${ }^{42}$. Plus encore, l'auteur met d'abord en scène l'univers symbolique à partir duquel se déploie la « relation de guérissage » et qui lui donne son sens. À partir de cette recherche, l'ethnomédecine de la Nouvelle-Calédonie ne pourra plus être la même, elle sort des raisonnements récurrents où elle était enfermée depuis des décennies. Mais, plus important pour la psychiatrie, dans son renouvellement, l'ethnomédecine appréhende une cosmogonie qui donne un sens et une logique aux discours kanak sur la maladie et ses causes, ne permettant plus les simplifications et les raccourcis diagnostiques psychatriques en termes de " pensée mythique », de délire et de persécution.

\section{L'infrastructure psychiatrique en Nouvelle- Calédonie}

L'hôpital psychiatrique Albert Bousquet est le pivot de la psychiatrie pendant plusieurs décennies. Nouville, comme on le nomme, est l'ancien hôpital du bagne sur l'île Nou, récemment rattachée à la Grande Terre par une route gagnée sur la mer. L'île Nou où seront construits les premiers bâtiments du bagne, dont le premier convoi arrive en 1864, est cédée par le colon santalier anglais James Paddon (dont une école primaire de la banlieue de Nouméa porte le nom) contre 4000 hectares confisqués de la chefferie du Grand chef Kuindo (Christnacht, 1990 : 12). Paddon est entré au panthéon des héros de la colonisation qui ont fait souche dans le pays.

En 1868 (Chuiton, 1979 : 1-2), un médecin de la marine, le docteur Lacroix, obtenait la construction de «l'hôpital du Marais » proche des locaux pénitentiaires dont il avait la charge médicale. L'hôpital comprenait cent soixante lits répartis dans quatre bâtiments reliés par une galerie. En 1879, l'hôpital devient « Dépôt de l'orphelinat », locaux disciplinaires pour une population indigène, océanienne et asiatique. En 1927, le Dépôt est transformé en Établissement d'Assistance Publique pour infirmes, vieillards, indigents et aliénés ainsi que pour des travailleurs étrangers et indigènes. En 1936, c'est la création de l'« Asile de Nouville » auquel est ajoutée, en 1941, une annexe de l'hôpital de Nouméa pour les malades immigrants et indigènes, ainsi qu'un camp de détention pour des ressortissants de "puissances ennemies » ou leurs « sympathisants ». En 1944, une réorgani- sation est opérée et, en 1952, l'Asile devient l'Hospice de Nouville. Les ateliers disciplinaires disparaissent ainsi que le camp de détention. En 1972, le rattachement de l'île Nou à la Grande Terre par une digue diminue son isolement. En 1978, l'établissement devient « Centre hospitalier spécialisé ».

Sur un site magnifique, les bâtiments carcéraux s'étendent jusqu'à la plage. Avec ses quartiers fermés et ouverts et sa petite route du bord de mer, l'hôpital oscille entre la villégiature et la prison. Comme il donne sur la plage, on ne peut s'en échapper que par la mer, car son porche est bien gardé. Ses grandes portes métalliques et son mur d'enceinte rappellent l'enfermement. Le médecin-directeur dont on a pris le nom pour le donner à cet endroit a été retrouvé mort sur la plage de l'hôpital un pistolet à la main en 1964. Dans un récent documentaire (Mignon, 1997), sa petite-fille réalisatrice évoque ce suicide occulté.

Depuis 1989, l'hôpital, auparavant géré par les militaires, est confié aux civils, il devient Établissement Publique Territorial et doit mettre en œuvre les orientations en matière de santé mentale votées par les élus du congrès du Territoire. Ces textes copient le plus souvent ceux de la lointaine métropole sans en avoir mis en œuvre les contenus en matière de désinstitutionnalisation, de décentralisation et de modernisation de la psychiatrie. Dans cet hors-lieu asilaire se côtoie une population bigarrée d'adultes. Ils sont souvent internés depuis de longues années. Dans la journée, c'est un endroit animé par le va-et-vient des patients, des visiteurs et du personnel qui travaille par roulement. Ces dernières années, l'hôpital a connu des mouvements de grèves, des conflits durs du personnel avec l'administration, des plaintes et des enquêtes administratives sur la qualité des soins, la gestion financière ${ }^{43}$ et beaucoup de difficultés à recruter des médecins psychiatres. Les transformations très importantes de ces dix dernières années favorisent un développement moins asilaire de la psychiatrie avec un recours moins systématique à l'hospitalisation, la création de plusieurs structures de soins et de consultations hors les murs, la fermeture des lits d'hospitalisation pour enfants et la création du service de pédopsychiatrie. La psychiatrie de secteur est en train de se développer, mais l'espace rural, moins bien desservi et sans infrastructures en santé mentale, ne bénéfice pas des mêmes développements.

42. Non pas que les différents ethnologues de la Nouvelle-Calédonie n'aient pas tenu compte du point de vue kanak, mais on le voit ici se déployer dans toute sa complexité et ses tâtonnements avec la présentation des propos des intéressés.

43. «Le CHS de Nouville épinglé par la chambre des comptes », article de presse du quotidien Les Nouvelles Calédoniennes, p. 4 du 17/11/1999. 


\section{Conclusion}

Jusqu'aux années 1990, psychiatres et psychologues en Nouvelle-Calédonie, à quelques exceptions près, se sont peu intéressés en terme de recherche à la population kanak et encore moins à celle résidant dans l'espace rural. Ils sont le plus souvent des expatriés en voyage, qui ont choisi de se fixer dans la colonie avec parfois comme corollaire une nécessité de déni, d'indifférence et d'ignorance de la population et de la culture autochtone. Détournant facilement à son profit les développements ethnopsychologiques discutables de l'œuvre de Maurice Leen- hardt, la psychiatrie coloniale produisait une théorisation de la personnalité kanak issue d'une clinique rudimentaire, en faisant l'économie de toute recherche en psychiatrie transculturelle et en ethnologie. Néanmoins, les transformations de l'anthropologie en Nouvelle-Calédonie, l'apport des linguistes et le renouveau de l'ethnomédecine ${ }^{44}$, conjugués aux transformations institutionnelles impulsées par le mouvement indépendantiste, contribuent à rendre progressivement caduque et obsolète les théorisations de la psychiatrie coloniale, en même temps qu'ils ouvrent la voie à de nouvelles pratiques de soins et à de nouveaux travaux de recherche.

\section{RÉFÉRENCES}

Angleviel Frédéric, 1994. Centenaire de Maurice Leenhardt, 1878-1954, Pasteur et Ethnologue, Nouméa, Publication de la Société d'Études Historiques de Nouvelle-Calédonie 52, I-XII.

Bensa Alban, 1995. Chroniques kanak, L'ethnologie en marche, Paris, Ethnies 10, 18/19.

Bourret Dominique et George Zeldine, 1978, La folie canaque, L'Évolution psychiatrique, 43, 3 : 549559.

Bourret Dominique, 1981-1982. Les raisons du corps. Éléments de la médecine traditionnelle autochtone en Nouvelle-Calédonie, Paris, Cahiers O.R.S.T.O.M. séries sciences humaines, XVIII, 4 : 487-513.

BRIL Blandine, 1997. Culture et premières acquisitions motrices : enfants d'Europe, d'Asie, d'Afrique, Journal de Pédiatrie et Puériculture, 3, 302-314, Paris.

Christnacht Alain, 1987 (mise à jour 1990). La Nouvelle-Calédonie, La documentation Française, Paris.

Chuiton Jacques, 1979. Historique du CHS de Nouville, Annexe de la plaquette du Centre Hospitalier Spécialisé (1991), «Bienvenue dans l'établissement », Nouméa.

Clifford James, 1980. Fieldwork, reciprocity and the making of ethnographic texts: the exemple of Maurice Leenhardt, Man, 15, 3 : 518-532.

_, 1987. Maurice Leenhardt, Personne et Mythe en Nouvelle-Calédonie, Paris, Jean Michel Place.

Collignon René, 1978. Vingt ans de travaux à la clinique psychiatrique de Fann-Dakar, Psychopathologie Africaine XIV, 2-3 : 133-141.
Corre A., 1894. L'Ethnographie Criminelle, Paris, Reinwald.

FRANCHETTE Éric, 1975, Introduction Ethnopsychiatrique, Thèse de Médecine, Université Paris VI.

GARELli Jacques, 1995. La phénoménologie du jugement et la dimension "cosmomorphique" du corps chez les Canaques, selon Maurice Leenhardt, Droit et Cultures, 29,1:255-274.

Guiart Jean, 1964. Maurice Leenhardt entre deux monde, Monde non chrétien, 71-72 : 193-197.

—, 1967. Du sorcier imaginé au voyant professionnel, Revue de l'Histoire des Religions, t172, 2 : 174-210.

ImMACOLATA OCCORSIO, 1988, La figure juridique de la congrégation des Sæurs missionnaires de la Société de Marie, à la lumière de ses origines et de l'évolution de son droit propre de 1857 à 1931, Rome, publication de l'Université Pontificale.

JoLly Margaret, 1991. To save the girls for brighter and better lives, Journal of Pacific History, 26, 1 : 27-48.

KUPER Adam, 1988. The invention of primitive society, London, Routledge.

LACHAUme, 1987. L'hystérie délirante canaque, L'Information Psychiatrique, 63, 10 : 1249-1257.

LAMBERT, 1900. Mours et Superstitions de Néocalédoniens, Publication de la Société d'Études historiques de la Nouvelle-Calédonie $n^{\circ} 14$, réédition 1985, Nouméa.

Laville-Cuenet Anne Françoise, 1988. Le vécu de la maladie mentale en milieu mélanésien de NouvelleCalédonie, Maîtrise en psychologie, Université de Toulouse le Mirail. 
LEENHARDT Maurice, 1931-33. Aurore d'âmes primitives, Monde non chrétien, $2: 29-52$.

_, 1944. Totem et identification, Revue de l'Histoire des Religions, 127 : 5-17, Paris, Puf.

_, 1947. Do kamo, Paris, Gallimard.

_, 1952. La propriété et la personne dans les sociétés archaïques, Journal de Psychologie Normale et Pathologique : 278-292.

LENORMAND Maurice, 1950. Connaissance du corps et prise de conscience de la personne chez les Mélanésiens de Lifou (Iles Loyalty), Journal de la Société des Océanistes, 6, $6: 33-66$.

LePoutre Marie, 1997. D’une médecine à l'autre, Thèse de Doctorat, EHESS, Marseille.

Mauss Marcel, 1960. Sociologie et Anthropologie, Paris, PUF.

Merle Isabelle, 1995. Expérience coloniale. La Nouvelle-Calédonie (1853-1920), Paris, Belin.

MÉTAIs Éliane, 1967. La sorcellerie canaque actuelle. Les tueurs d'âmes dans une tribu de la NouvelleCalédonie, Paris, Publication de la Société des Océanistes $\mathrm{n}^{\circ} 20$.

Mignon Mathilde, 1997. Folles mémoires d'un caillou, film, France, Production la Sept Arte, les Films d'ici, Simple Production, RTBF, RFO.

De Muolla Marie-Cécile, 1980. Les pionnières maristes en Océanie, aux origines des sæurs missionnaires de la Société de Marie 1845-1931. Rome, Tipografia Artistica.

Montandon Georges, 1937. Les races du monde océanien, Bulletin de la Société des Océanistes, 1 : 11-32.

Moro Marie-Rose, 1998. Psychothérapie transculturelle des enfants de migrants, Paris, Dunod.

MouchenIK Yoram, 2000. L'enfant vulnérable, paroles, récits et représentations familiales de l'enfant dans une relation psychothérapique sur les îles d'Ouvéa et de Maré dans l'archipel des Loyauté en Nouvelle-Calédonie, thèse de Doctorat, EHESS, Paris.

NAEPEls Michel, 1996. Conflits fonciers et rapports sociaux dans la région de Houaïlou (NouvelleCalédonie), thèse de Doctorat, EHESS, Paris.

_, 1998. Histoire de terres kanakes. Conflits fonciers et rapports sociaux dans la région de Houaïlou (Nouvelle-Calédonie), Paris, Belin.
Nos pionnières, Sœurs, missionnaires de la Société de Marie d'après la correspondance, 1836-1885, quatre volumes, 1973,1975, Administration générale, Rome.

Pales Léon, Claude Chippeaux et Henri Pineau, 1960. Le pied dans les races humaines. Les Mélanésiens de la Nouvelle-Calédonie et des îles Loyauté, comparés aux Français, Malgache, MélanoAfricains et Vietnamiens, Journal de la Société des Océanistes, Tome XVI, ${ }^{\circ} 16$, déc. : 45-90.

Poinso F. et C. Vedié, 1991. Étude statistique des maladies mentales en Nouvelle-Calédonie, Société Médico-psychologique : 663-673.

Porot Antoine, 1943. L'œuvre psychiatrique de la France aux colonies depuis un siècle, Annales Médico-psychologiques : 356-378, t 1.

PozNANSKi Renée, 1994. Etre juif en France pendant la Seconde Guerre mondiale, Paris, Hachette.

DE Rochas Victor, 1860. Notes sur le délire aigu chez le Néo-Calédonien, Bulletin de la Société de Biologie, Paris.

SAlomon Christine, 1993. Savoirs, savoirs-faire et pouvoirs thérapeutiques : guérisseurs Kanaks et relation de guérissage dans la région centre nord de la Grande Terre, A.D.C.K. Nouméa.

—, 1999. Côté femmes : la maternité, une exigence sociale maintenue, le contrôle sur la reproduction, une marge d'autonomie confisquée, in Chronique $d u$ pays kanak, tome 2 : 188-195, Nouméa, Planète mémo.

—, 2000. Savoirs et pouvoirs thérapeutiques kanaks, Paris, Puf.

SARASIN Fritz, 1917. La Nouvelle-Calédonie et les Iles Loyauté, Souvenirs de voyage d'un naturaliste, Bâle/Paris, Fischbacher.

SElod Sophia, 1998. Difficultés diagnostiques chez les patients d'une autre culture: pourquoi certains patients dépressifs sont-ils diagnostiqués comme psychotiques? Thèse de Doctorat d'État, Faculté de Médecine Lille2, Lille.

VInson Eugène, 1858. Éléments d'une Topographie Médicale de la Nouvelle-Calédonie et de l'Ile des Pins, Thèse de Médecine, Paris.

ZELDINE Georges, 1981. Sociologie des maladies mentales en Nouvelle-Calédonie (Approche statistique), Annales Médico-Psychologiques: 385-408, Vol 139, 4. 